\title{
Fine Needle Aspiration of Glomangioma Knee - A case report
}

\author{
Syed Besina Yasin, M.D., Sumyra Khurshid Qadri, M.D., Nassima Chanda, M.D., Rumana Hamid \\ Makhdoomi, M.D. \\ Department of Pathology, Sher-i- Kashmir Institute of Medical Sciences (SKIMS), Srinagar (J\&K), India
}

\section{A B S T R A C T}

Glomus tumor, a benign neoplastic proliferation of modified smooth muscle cells characteristically occurs in a digital subungual location and presents with localised pain. Extra-digital locations that have been described so far include bone, tongue, stomach, rectum, lung, mediastinum, sacrum, coccyx and head and neck areas. Though the histology of glomus tumor is very characteristic, cytological features are poorly defined. Less than ten cases describing cytologic features have been reported so far. We present a case of glomus tumor of the knee joint detected on fine needle aspiration and confirmed later on histopathology, in a 55-year-old male who presented with history of painful nodule on the anterior aspect of the left knee joint for one year. JMS $2011 ; 14(2): 71-73$

Key words: Glomangioma, fine needle aspiration cyłology

Glomus tumor is an unusual tumor comprising 1-2\% of soft tissue tumors ${ }^{1}$. It is a benign neoplastic proliferation of modified smooth muscle cells. ${ }^{2}$ A normal glomus is a specialised form of arterio-venous anastamosis that is responsible for blood and temperature regulation. ${ }^{3}$ The lesion characteristically occurs in a digital subungual location and presents with well localised pain that is exacerbated by temperature change. ${ }^{2}$ These tumors have been described in extra digital locations such as bone, tongue, stomach, rectum, lung, mediastinum, sacrum, coccyx and head and neck areas. Though the histology of glomus tumor is very characteristic, cytological features are poorly defined. Less than ten cases describing cytologic features have been reported so far. We herein present a case of glomus tumor on the anterior aspect of the knee joint detected on fine needle aspiration and confirmed later on histopathology.

Correspondence and reprint requests to:

Dr. Syed Besina Yasin,

Associate Professor,

Department of Pathology,

Sheri-i-Kashmir Institute of Medical Sciences, Srinagar.

Email: drsyedbesina@yahoo.com

\section{Case report}

A 55-year-old male presented with history of painful nodule on the anterior aspect of the left knee joint for past one year. On physical examination, there was a small $0.5 \mathrm{~cm}$ subcutaneous nodule. It was reddish-blue in colour, mobile, firm and was intensely painful on touch. Routine investigations were within normal limits. A clinical diagnosis of neurofibroma or glomangioma was made. Fine needle aspiration cytology (FNAC) was performed using a $20 \mathrm{ml}$ syringe with 22 gauge needle. The patient experienced excruciating pain during the procedure. A blood-mixed material was obtained. Smears were stained with Papanicolaou and May-Grunwald Giemsa stains. On microscopic examination, the aspirate was heavily admixed with blood. There were cohesive clumps of uniform round to oval cells along with wisps of magenta coloured intercellular material. The nuclei were round to oval, with firmly distributed chromatin. Intranuclear inclusions, nucleoli and mitosis were not seen. Cytoplasm was poorly defined (Figure 1). A few groups of spindle shaped cells were seen near the clusters of tumour cells. Numerous RBC's were seen in the background. A diagnosis of glomangioma was offered on FNAC based on the clinical features and the cytologic features. 


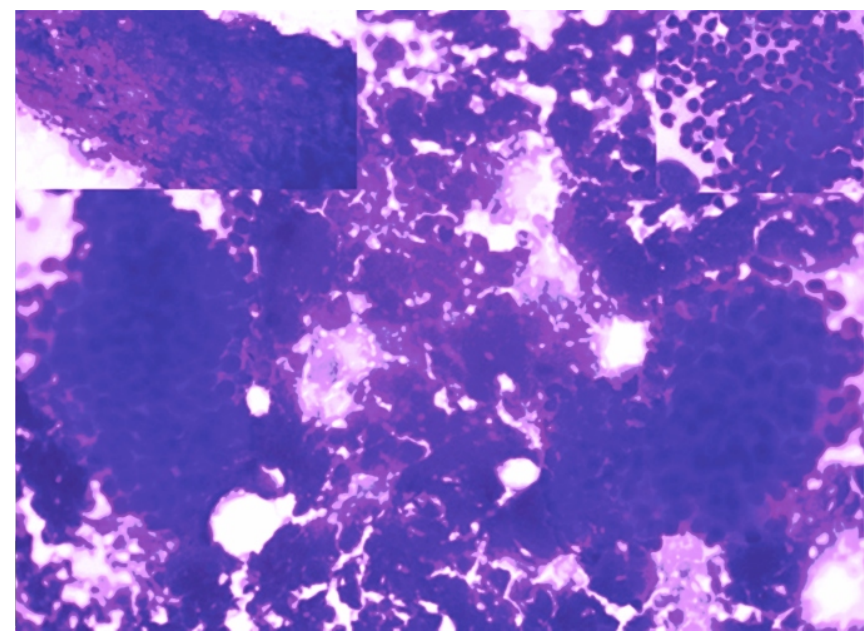

FIGURE 1: FNAC smear showing two cohesive clumps of uniform round glomus cells; Right inset showing a clump of glomus cells; Left inset showing intercellular material with entrapped endothelial cells. (MGG, x 400)

The lesion was excised and gross examination revealed a small nodule $0.5 \times 0.5 \mathrm{~cm}$ in size. The cut surface was greyish white. Microscopy revealed a well circumscribed tumor consisting of uniform round to oval cells in nests and clumps around thin walled capillary sized blood vessels which were lined by endothelial cells. Cells showed a uniform central rounded nucleus with mild to moderate eosinophilic cytoplasm (Figures $2 \& 3$ ). PAS and reticulin stain demonstrated a dense reticulin network investing the single tumor cells. Based on all the above features, a diagnosis

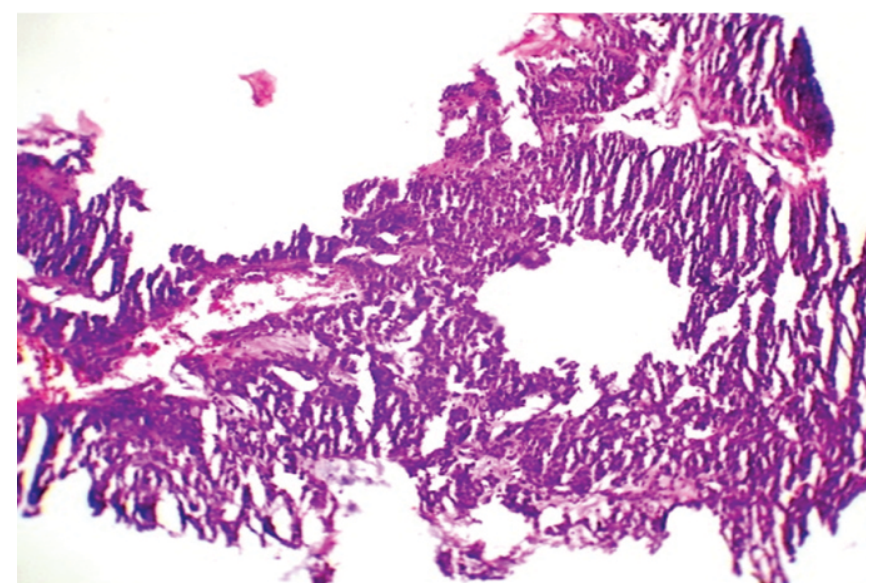

FIGURE 1: FNAC smear showing two cohesive clumps of uniform round glomus cells; Right inset showing a clump of glomus cells; Left inset showing intercellular material with entrapped endothelial cells. (MGG, x 400)

of glomus tumor was given. Postoperatively, the patient was completely relieved of pain.

\section{Discussion}

Glomus tumors are uncommon tumors with an estimated incidence of $1.6 \%$ among the 500 consecutive soft

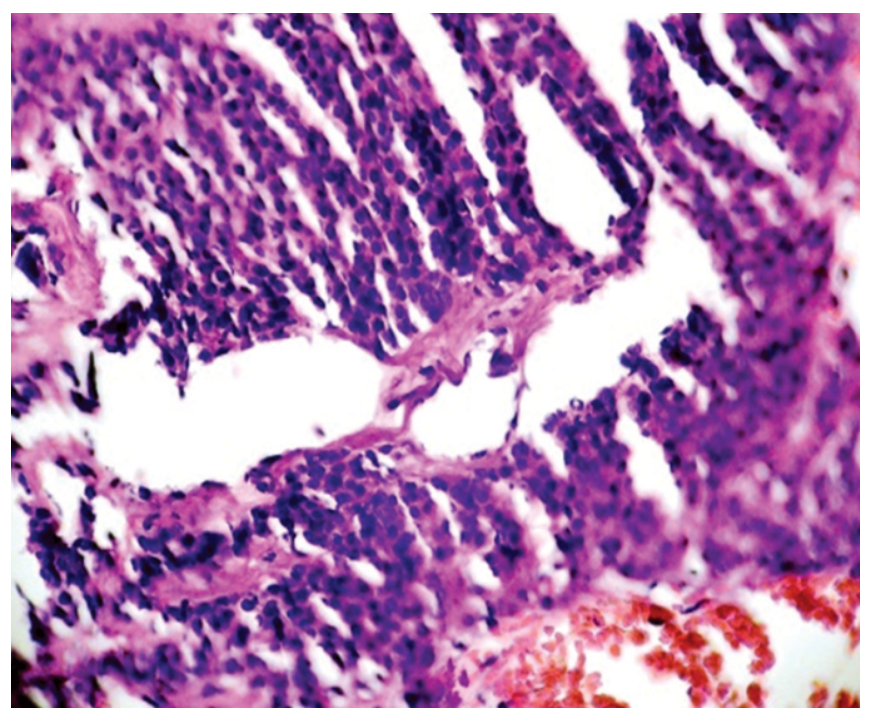

FIGURE 3: Photomicrograph showing uniform round to oval cells in nests and clumps around thin walled blood vessels lined by endothelial cells. (H \& E, x 400)

tissue tumors reported from the Mayo Clinic. ${ }^{4}$ They are composed of cells that resemble the modified smooth muscle cells of a glomus body. ${ }^{5}$ Normal glomus cells are located in the subcutaneous tissue which is responsible for the regulation of temperature and blood pressure. Glomus tumors may be single or multiple. Usually, single tumors are idiopathic and multiple ones are inherited as an autosomal dominant trait. ${ }^{3}$ The tumor is about equally common in both genders, although there is a striking female predominance among patients with subungual lesions. Most glomus tumors are diagnosed during adult life. ${ }^{4}$ The classic triad of aching pain, exquisite tenderness and temperature sensitivity provide the diagnosis. ${ }^{3}$ The most important site is the subungual region but other common sites include palm, wrist, forearm and foot. ${ }^{4}$ Rare sites include stomach, mediastinum, lung, trachea, vagina, etc. ${ }^{1}$

Our patient was a 55 year male with a solitary $0.5 \mathrm{~cm}$ diameter subcutaneous nodule on the anterior aspect of the left knee which is a less common site. Patient presented with aching pain and extreme tenderness. A clinical diagnosis of glomus tumor/ neural tumor was offered. There was no clue from the radiological investigations to confirm the diagnosis. FNAC was done. A diagnosis of glomus tumour was suggested on the basis of typical features on FNAC and later confirmed on HPE of the excised tumour mass.

Less than ten cases of this tumor have been reported on fine needle aspiration. ${ }^{1}$ Cytological features of this tumor were first described by Holck and Bredeson in 1996. They observed that the aspirated smears were cellular, composed of tight clusters and more loosely packed sheets of medium sized polygonal cells as well as frequent stripped nuclei. At places, the cell groups form a 3-dimensional pattern. The nuclei were round to oval with finely distributed chromatin. Intranuclear inclusions and nucleoli were inconspicuous as were mitosis. The cytoplasm was poorly defined. Rows of spindle cells, probably endothelial cells, focally crisscrossed 
the cell groups. At places delicate feathery, curved threads of reddish material corresponded to myxoid substance and basement membrane material was seen. ${ }^{6}$

Vidyavathi et al, reported a glomus tumor on fine needle aspiration of a 28-year-old female with a $2 \times 1 \mathrm{~cm}$ mobile swelling in the forearm. ${ }^{1}$ Endoscopic ultrasound guided fine needle aspiration of gastric submucosal tumors has revealed one glomus tumor in a study conducted by Sawaki et al, on 304 lesions. Vinette and Yazdi have also reported a glomus tumor of stomach on fine needle aspiration in a 72-year-old asymptomatic female. ${ }^{8} \mathrm{M}$ Perez-Guillermo et al, in a study of 12 cutaneous vascular tumors by means of fine needle aspiration, reported one glomus tumor ${ }^{1}$. The differential diagnosis includes cutaneous adnexal tumors like eccrine spiradenoma, carcinoid tumor or a hemangiopericytoma. ${ }^{8}$ Due to location, adnexal tumor was the differential diagnosis in our case. Cytologic features of glomus tumor and eccrine spiradenoma which form close differential diagnosis have been rarely reported. Smears of eccrine spiradenoma show presence of bland uniform cells in cohesive clusters and cribriform sheets with rosette-like structures surrounding amorphous material. Three types of cells are also seen-large epithelial cells, myoepithelial cells and smaller lymphocytes. ${ }^{1}$ All these features were not seen in our case.

The presence of excruciating pain on aspiration and cohesive clusters of small round cells with presence of basement membrane like magenta-coloured material along with groups of spindle shaped endothelial cells lead to the diagnosis of glomus tumor.

The histologic features of glomus tumor have been well described in literature. On gross examination, they are blue or purple and less than one centimetre in size. Microscopically, the glomus tumors are relatively well circumscribed capsulated lesions and show numerous small vascular lumina surrounded by clusters of monotonous polygonal glomus cells set in a hyalinised or myxoid stroma. The glomus cells are characterised by a faintly eosinophilic cytoplasm and large, punched out pale nuclei with delicate chromatin and inconspicuous nucleoli.' On Immunohistochemistry, vimentin and SMA can be identified in nearly all glomus tumors. Desmin is highly variable. ${ }^{4}$

In conclusion, it is emphasised that in addition to neural tumors and adnexal lesions like eccrine spiradenoma, glomus tumors should also be considered in the differerntial diagnosis of all soft tissue tumors associated with pain and tenderness. Fine needle aspiration is a good tool at arriving the correct diagnosis in such cases.

\section{References}

1. Vidyavathi K, Udayakumar M, Prasad CS BR, Harendra Kumar ML. Glomus tumor mimicking eccrine spiradenoma on fine needle aspiration. $J$ of Cytology 2009; 26(1):46-48.

2. Perks JF, Beggs I, Lawson MG, Davie R. Juxtacortical glomus tumor of the distal femur adjacent to the popliteal fossa. Am J of Roentgenology 2003;181:15901592.

3. Rathi KR, Jena L, Dash BM, Metra D, Patnaik PK, Basu AR. Extradigital glomus tumor as a cause of chronic perianal pain. Indian J of Pathol and Microbiol 2009; 52(3):414-416.

4. Enzinger FM and Weis SW. Perivascular tumorss In: (Soft tissue tumors) Eds. (Weis SW \& Goldblun JR, 5th Edition 2008, USA:751-767.

5. Oscar Lin and Maureen F Zakowski. Cytology of soft tissue, Bone and skin. In Comprehensive Cytopathology. 3rd edition eds. Bibbo M and Wilbur DC. Saunder Elsevier 2008: 479-480.

6. Holk S, Bredeson LP. Solid glomus tumor presenting as axillary mass. Report of a case with morphologic study, including cytologic characteristics. Acta Cytol 1996;40: 555-562.

7. Sawaki A, Misuno N, Hoki N, Takagi T, Nakamura T, Tajika $\mathrm{M}$ et al. Diagnosis of gastric submucosal tumors using endoscopic ultrasound guided fine needle aspiration.J Clin Oncol 2008;26:20.

8. Sapi Z, Antal I, Papai Z, Szendroi M, Mayer A, Jakab K, et al. Diagnosis of soft tissue tumors by fine needle aspiration with combined cytopathology and ancillary techniques. Diagnostic Cytopathology 2002;26(4);232242.

9. Guillermo-Perez M, Solar Perez B, Rojo G, Gil H. Fine needle aspiration cytology of cutaneous vascular tumors. Cytopathology 2007;3(4):231-244.

10. DN Slater, DWK Cotton, Azzopardi JG. Oncocytic glomus tumor: a new variant. Histopathology 1987;11: 523-531.

11. Vinette-Ledue D, Yazdi HM. Fine needle aspiration biopsy of a glomus tumor of the stomach. Diagnostic Cytopathology 2001;24(5):340-342. 